$10-2005$

\title{
Analysis of gaits for a rotating tripedal robot
}

Damian M. Lyons

Fordham University

Kiran Pamnany

Fordham University

Follow this and additional works at: https://fordham.bepress.com/frcv_facultypubs

Part of the Robotics Commons

\section{Recommended Citation}

Lyons, Damian M. and Pamnany, Kiran, "Analysis of gaits for a rotating tripedal robot" (2005). Faculty Publications. 30. https://fordham.bepress.com/frcv_facultypubs/30

This Article is brought to you for free and open access by the Robotics and Computer Vision Laboratory at DigitalResearch@Fordham. It has been accepted for inclusion in Faculty Publications by an authorized administrator of DigitalResearch@Fordham. For more information, please contact considine@fordham.edu. 


\title{
Analysis of gaits for a rotating tripedal robot
}

\author{
Damian. M. Lyons and Kiran Pamnany \\ Robotics \& Computer Vision Laboratory \\ Fordham University \\ NY 10458 \\ dlyonsecis. fordham.edu
}

\begin{abstract}
A goal of robotics has been to develop mechanisms that have the efficiency and speed of wheeled robots with the terrain flexibility of legged robots. In previous work, we have proposed a unique three-legged mechanism, the rotopod, designed to integrate these two useful approaches to locomotion.

In this paper, we present an analysis of the variety of gaits that can be exhibited by the rotopod. We present examples of many of these gaits and discuss their potential use. A trajectory generation algorithm is presented that can be used to generate point to point trajectories using one of three different styles of gait.
\end{abstract}

\section{Introduction}

One of the most striking capabilities of animals in general is their ability to orient to a potential danger and to evade it at high speed if necessary. The erratic zig-zag of a surprised vole, or the almost instantaneous disappearance of a fish when a pebble hits the water, leaves a strong impression of speed and maneuverability. The blend of perception and locomotion abilities required to do this is not something that can be easily reproduced with current robotic technology. The rotopod mechanism [6,7] consists of three legs arranged in a tripod fashion. Each leg has a single translational degree of freedom. A single rotating joint is mounted at the apex of the legs, rotating parallel to the ground plane. The joint continuously rotates a reaction mass mounted at the end of a lever (see Fig. 1). By retracting the legs in turn, the rotating reaction mass can be used to swing the platform around each of the retracted legs. This robot has the advantage of legged locomotion in stepping its 1-DOF legs over objects, but its drive mechanism is a rotating reaction mass that rotates the robot, in a controllable fashion, around each of its legs, similar to a rotating wheel. The mechanism has the potential to transfer the energy from the rotating reaction mass in an efficient manner to the legs, effecting a spinning forward motion. Such a device could have applications as a small fast-moving and extremely agile mobile sensor platform. A large quantity of small versions of this mechanism could be dropped to map and explore an area, or larger versions could be used as for unmanned forward reconnaissance.

In [6] we have described the rotopod mechanism and how it can be moved purposefully. [7] builds on this to look at a selection of gaits for the mechanism and introduces a simple power model. In this paper, after a brief review of the mechanism and the method of stepping, we present a taxonomy of gaits for the rotopod, and outline an approach to trajectory generation that takes advantage of the strengths of the unique platform. We conclude with some simulation results for trajectory generation.

\section{Literature Review}

The literature has included many examples of wheeled and legged robots, and the relationship between them has been well studied. Wheels offer fast and energy efficient travel, whereas legs allow transit over difficult terrain. Both of these capabilities are highly desirable, and researchers have attempted to combine legs and wheels within a single platform, resulting in many different platform designs. Halme et al.'s WorkPartner [3] has wheels at the end of its legs. Case Western's cricket-inspired robot [1] has wheels in the

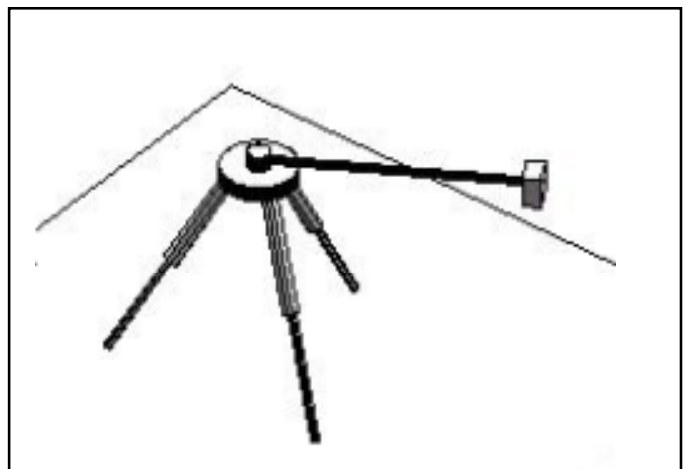

Figure 1: The Rotopod Mechanism 
front and legs in the back. The Univ. of Michigan's RHex [9] has four legs that are single-spokes - legs - mounted on axles, and Case Western's Whegs robots [8] have six legs that are each 3 spokes on axles. The research leading to the Whegs robot, for example, was biologically inspired; the platform itself is biomimetic in copying the six legged insect configuration, but departs in that it includes a rotating axle element for each leg. This is a uniquely efficient mix of biology and engineering.

Recently, we have developed a unique class of mechanism that combines the capabilities of wheeled and legged robots [6]. The mechanism is not biomimetic, however, the unique variety of locomotion options it offers means that it is an exciting platform upon which to build a robust intelligence that can operate in an unstructured environment.

\section{The Rotopod Mechanism}

The rotopod consists of three legs arranged in a tripod configuration. Each leg has a single translational degree of freedom ${ }^{1}$. A rotational degree of freedom at the apex rotates a reaction mass attached to an arm as shown in Fig. 1. In a plan view (Fig. 2(a)) the legs make an angle of $120^{\circ}$ with each other. In a side view where 2 legs line up, the angle the third leg makes with the vertical centerline is $\beta$ ( $45^{\circ}$ in our prototype). Each leg has an extended length $l_{e}=l+\delta$ where $l$ is the retracted length of the leg. The distance along the ground from the end of a leg to the ground projection of the apex is $l_{e} \operatorname{Sin} \beta$.

When one leg retracts, the plane of the rotating reaction mass is no longer parallel to the ground (Fig. 3); call the resultant angle of tilt $\alpha$. The retracted leg drops a distance $\delta \operatorname{Cos} \beta$. The platform tilts by rotating around the line joining the two extended legs. From this, the angle of tilt can be calculated as:

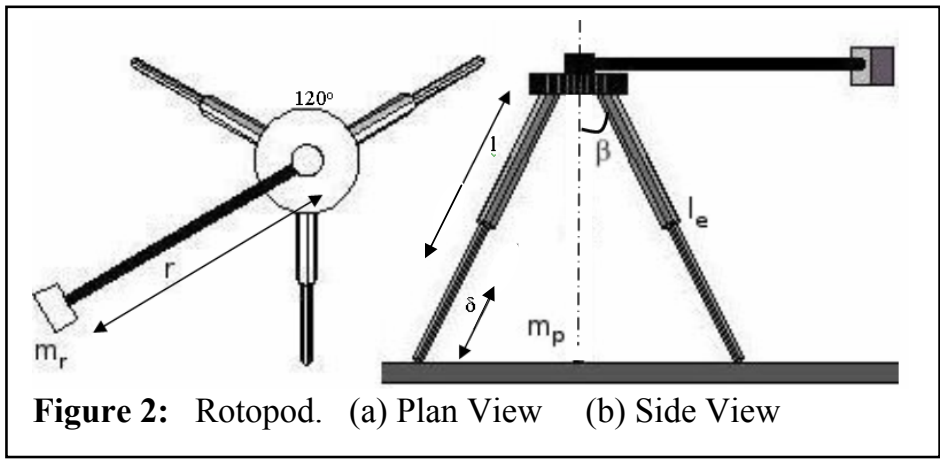

$$
\tan \alpha=\frac{\cot \beta}{1.5\left(l_{e} / \delta\right)-1}
$$

When the reaction mass is rotating, the platform experiences a force radially outward along the arm. If the arm has an angular velocity $\omega$ then the force is given by $f_{r}=m_{r} \omega^{2} r$. The force is opposed by the weight of the platform $f_{w}=m_{p} g$. When the platform is tilted, and the reaction mass is directly over the retracted leg, these forces can be resolved along the retracted leg. $f_{r}$ will have a component that causes a moment attempting to rotate the retracted leg (Fig. 3) clockwise about its endpoint, while $f_{w}$ will have a component that causes a moment that rotates the leg counterclockwise. The net moment will be ${ }^{2}$ :

$$
M_{n e t}=f_{w} l_{e} \operatorname{Sin}(\beta-\alpha)-f_{r} l_{e} \operatorname{Cos} \beta
$$

Note that $M_{\text {net }}$ will increase as the arm approaches and travels over a leg, and decreases as it moves away, proportional to the cosine of the angle the arm makes with the leg. Let us consider the case where the arm is directly over the leg. If $M_{n e t}$ is negative, the moment will be countered by the reaction forces supplied by the other legs. If it is positive, then the platform will begin to unbalance, tilting over the retracted leg, and pulling the other two legs off the ground. By choosing $\alpha$ (and/or $\omega$ ) sufficiently large the platform can be driven to this state. In our existing prototype, we keep $\omega$ constant and vary $\alpha$ by retracting or extending the legs.

In the case that the other two legs lift off the ground, then

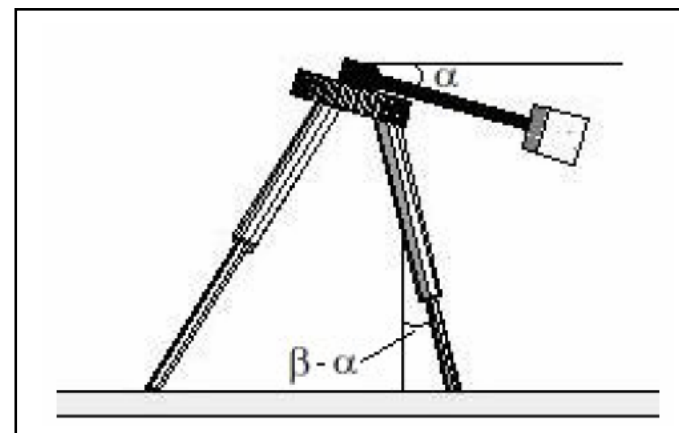

Figure 3: One Leg Retracted

${ }^{1}$ The prototype in [6] has a single rotational degree of freedom per leg.

${ }^{2}$ This assumes that the center of gravity is under the apex and does not vary as the reaction mass moves; not strictly true, but the expressions become less transparent when this is added. 


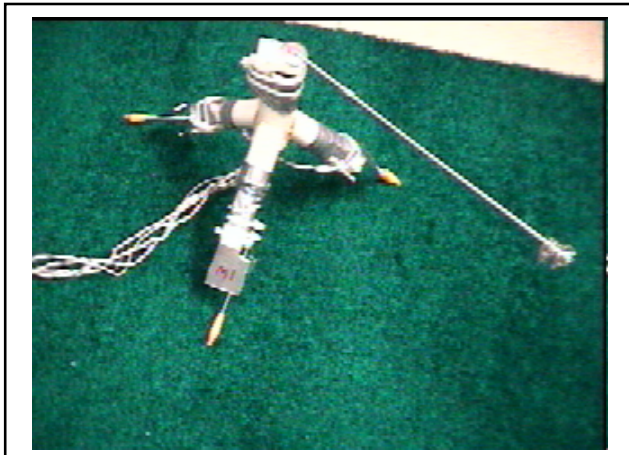

Figure 4: Prototype Platform

\begin{tabular}{||l|l|l|}
\hline Name & Description & Value \\
\hline$\ell 1$ & Length of lower leg & $10.2 \mathrm{~cm}$ \\
\hline$\ell 2$ & Length of upper leg & $10.2 \mathrm{~cm}$ \\
\hline$\ell 3$ & Vertical offset of reaction mass joint & $8.9 \mathrm{~cm}$ \\
\hline$\ell 4$ & Length of reaction mass arm & $30.5 \mathrm{~cm}$ \\
\hline$\ell_{\mathrm{e}}$ & Extended Leg length $(\ell 1+\ell 2)$ & $20.3 \mathrm{~cm}$ \\
\hline & Min/Max angle of leg bend & $0^{\circ} /+30^{\circ}$ \\
\hline$\beta$ & Angle between leg and centerline & $45^{\circ}$ \\
\hline $\mathrm{m}_{\mathrm{r}}$ & Reaction mass & $30 \mathrm{~g}$ \\
\hline $\mathrm{m}_{\mathrm{v}}$ & Vehicle mass without $\mathrm{m}_{\mathrm{r}}$ & $430 \mathrm{~g}$ \\
\hline $\mathrm{m}$ & Total mass $\left(\mathrm{m}_{\mathrm{r}}+\mathrm{m}_{\mathrm{v}}\right)$ & $460 \mathrm{~g}$ \\
\hline
\end{tabular}

Table 1: Prototype Parameter Values

the torque of the rotating apex joint no longer has a reaction torque from those legs, and the platform will rotate around the retracted leg endpoint. This rotation moves the center of the rotopod a distance proportional to the angle rotated, $\theta$. Once the retracted leg is then extended again, the net moment is reduced, and the other legs are lowered to the ground, and the platform is brought back into balance.

The angle $\theta$ can at most be $\omega t$ where $t$ is the time between retraction and extension of the leg. In practice, $\theta$ is much less than this, due to friction in the apex joint and at the leg endpoint. (This friction also means that the reaction mass will move relative to the retracted leg while the platform is unbalanced, reducing the positive net moment and bringing the platform back towards balance, hence placing a limit on the amount of time the platform can be unbalanced; giving a natural tendency to stability.) Furthermore, when the leg is again extended, some rotational slip may occur at the endpoints of the other leg. These factors make the relationship between $\omega t$ and $\theta$ difficult to model accurately. In [6] we proposed a linear relationship, $\theta=\eta \omega t$ and we measured $\eta$ empirically. We then controlled the stepping angle $\theta$ by controlling the duration a leg was retracted.

The prototype used in these experiments is shown in Fig. 4. The body consists of four hollow structural members, three forming a bottom tripod, and one extending from the apex of the tripod. DC motors with gearboxes were mounted within each of the leg members, and a light metal rod attached to the gearbox output axle to serve as a lower leg. Two limit stops were added on each leg to limit the lower leg rotation to the range $0^{\circ}$ to $30^{\circ}$ with respect to the upper leg. A DC motor was also mounted in the apex member and a longer light metal rod was attached to the gearbox axle to serve as a lever arm for the reaction mass. The reaction mass consisted of one or more u-bolts. Table 1 shows the parameter values for the prototype.

Fig. 5 shows one graph of the data used to

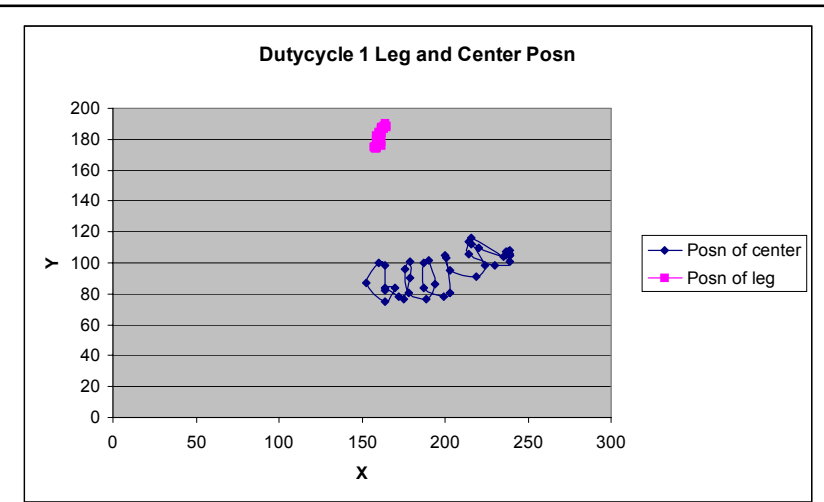

Figure 5: Graph of leg \& center positions during motion

evaluate $\eta$. An average value of 0.55 was obtained from these experiments. Note the epicycloidal motion of the center during the step in Fig. 5. This was due to passive movement of the leg joints as the reaction mass rotated. It could be eliminated by active stiffening of the legs, but it has not impaired performance and correcting it would use additional power.

\section{Rotational Gaits}

We identify two modes of stepping. In static stepping, the rotopod brings itself back into a statically stable $\left(M_{\text {net }} \leq 0\right)$ configuration at the end of each step, as described above. In dynamic stepping, the rotopod remains in an unbalanced configuration between rotations. We will restrict our attention to static stepping in this work. In this case, we see that 
on each step, the rotopod can rotate for a different angle around each of its legs. The rotation around a single leg can be captured by a combined rotation and translation $R T$ as follows:

$$
R T(L, \theta)=\left[\begin{array}{ccc}
\operatorname{Cos} \theta & -\operatorname{Sin} \theta & L_{x} \operatorname{Cos} \theta-L_{y} \operatorname{Sin} \theta-L_{x} \\
\operatorname{Sin} \theta & \operatorname{Cos} \theta & L_{x} \operatorname{Sin} \theta+L_{y} \operatorname{Cos} \theta-L_{y} \\
0 & 0 & 1
\end{array}\right]
$$

where $L=\left(L_{x}, L_{y}\right)$ is the position of the leg relative to the center in a plan view. The velocity of the center can be related to the angular velocity $\omega$. For a single rotation,

which can be rewritten as

$$
v=\frac{d(R T(L, \theta))}{d t}=\frac{d(R T(L, \theta))}{d \theta} \frac{d \theta}{d t},
$$

$$
v=\left[\begin{array}{ccc}
0 & -\eta \omega & 0 \\
\eta \omega & 0 & 0 \\
0 & 0 & 1
\end{array}\right] R T(L, \theta)
$$

If $p_{c}$ is the center position, then after a rotation of $\theta 1$ around $\mathrm{L} 1, \theta 2$ around $\mathrm{L} 2$ and $\theta 3$ around $\mathrm{L} 3$, the new position is

$$
p 1_{c}=R T(L 3, \theta 3) R T(L 2, \theta 2) R T(L 1, \theta 2) p_{c}
$$

We call a repeated pattern of rotations a gait and we write a gait as a list of (leg, angle) pairs. The rotopod mechanism can be moved in a variety of gaits. For convenience we group them according to the following taxonomy:

1. Simple Gaits: The gait occurs within a single rotation of the reaction mass.

2. Compound Gaits: The gait takes more than one rotation of the reaction mass.

\subsection{Simple Gaits.}

These are gaits whose periods occur within a rotation of the reaction mass.

1. Single Leg Gaits $g l=[(L i, \theta)] i \in\{1,2,3\}$ : rotation around a single leg. This is the simplest kind of motion for the mechanism. Figure 6 shows an overhead view of the motion of a rotopod with the single leg gait $g l=\left[\left(L 1,20^{\circ}\right)\right]$ for 3 repetitions of the gait. The rotopod is shown in a different shade for each repetition of the gait, and a small dot is shown at the end of the leg around which rotation occurs. The black line joining the center of the tripods shows the path of the center of the mechanism. The center $p_{c}$ will rotate in a circle of radius $l_{e} \operatorname{Sin} \beta$ around the end point of $L 1$ returning eventually to its original position. Figure 5 shows a trace of the prototype mechanism performing a similar gait.

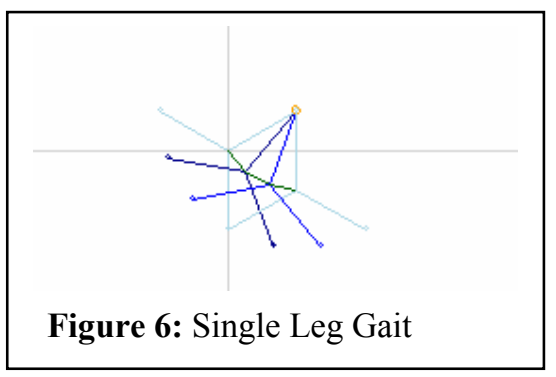

2. Two Leg Gaits $g 2=[(L i, \theta 1),(L j, \theta 2)] i, j \in\{1,2,3\}, i \neq j$ : rotation around two (successive) legs. A single leg gait can only effect a circular motion of $p_{c}$; however, the addition of rotation around a second leg produces a more complex motion. Fig. 7 (a) through (j) show examples of two leg gaits; all are on legs 1 and 2. The effect of switching legs does not change the pattern produced, just its relation to the starting position. Fig. 7(a)-(f) show equal angle gaits, and (g)-(j) show unequal angle gaits, and the number of gait repetitions is shown prefixed by an "x." Fig. 7(a) shows successive robot positions in different shades, but the others do not. All of these gaits if repeated sufficiently often will produce a closed path for $p_{c}$. These motion patterns will tend to cover the same area over and over. Some gaits will cover the ground more closely (e.g., 7(j)) than others (e.g., 7(c)), some will cover a larger area (e.g. 7(e)) than others (e.g., 7(h)). The parameters of the gait can be set to control the shape of a (typically annular) search area, and the number of repetitions controls the resolution of the search (See also Fig. 8 below) of that area. 
(a)

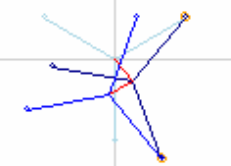

$20^{\circ} / 20^{\circ} \times 1$

(b)

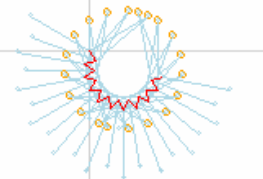

$20^{\circ} / 20^{\circ} \times 10$

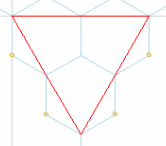

(f)
$120^{\circ} / 120^{\circ} \times 3$

(g)

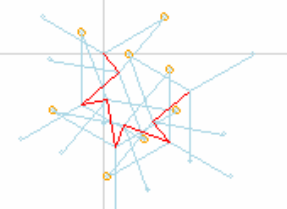

(h) (c)

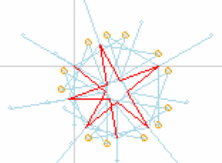

$40^{\circ} / 40^{\circ} \times 6$

(d)

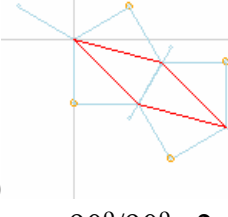

$90^{\circ} / 90^{\circ} \times 2$

(e)

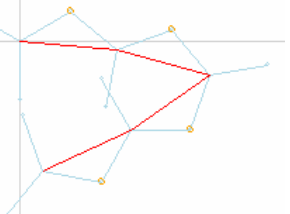

$110^{\circ} / 110^{\circ} \times 2$

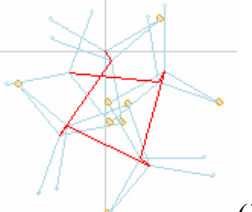

$10^{\circ} / 90^{\circ} \times 4$

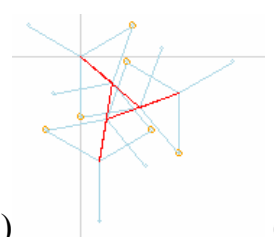

(i)

$40^{\circ} / 80^{\circ} \times 3$ (j)

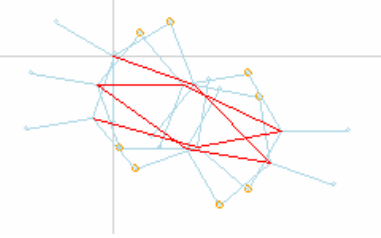

$80^{\circ} / 110^{\circ} \times 4$

Figure 7: Examples of Two Leg Gaits

Two leg gaits have the following potential applications therefore: (1) to produce spot maneuvers such as sharp turns (e.g., 7(e)), (2) to implement ground coverage (search, surveillance) applications, (3) to produce more energy efficient straight line motion (e.g., 7(f)) for short durations, or curved motion for longer durations, than 3 leg gaits (see next section).

3. Three Leg Gaits $g 3=[(L 1, \theta 2),(L 2, \theta 2),(L 3, \theta 3)]$ : rotation around all three legs. We can investigate the three leg gaits in a fashion similar to the two leg gaits. For example, Fig. 8 below shows a three leg gait used to search an annular area in increasingly refined steps. However, one new, very important mode of locomotion becomes possible with three leg gaits: straight line motion. When the mechanism rotates $120^{\circ}$ each leg exactly replaces the previous one (for

(a)

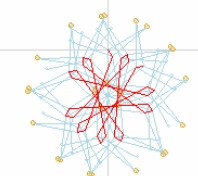

$10 \%$ (b)

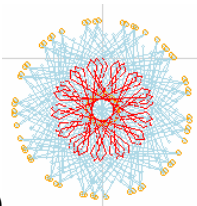

$10^{\circ} / 119^{\circ} / 10^{\circ} \times 30$ (c)

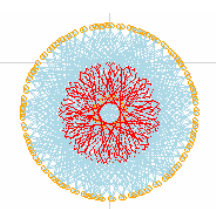

$10^{\circ} / 119^{\circ} / 10^{\circ} \times 70$
Figure 8: Three leg gait in increasingly refined search pattern a rotopod with more than three legs, this angle would be less of course) producing a straight line motion (Fig. 9(a)). If each leg rotates a little less than $120^{\circ}$, the mechanism will follow a circular path to the right; a little more than $120^{\circ}$, the circular path will be towards the left (Fig. 9(c),(d)). The radius of curvature $r_{c}$ for the circular path can be related to the angle rotated by each leg by

$$
r_{c}=\frac{d(\theta)}{2 \sin \frac{\gamma}{2}}
$$

where $\gamma$ is the deviation of the leg rotation $\theta$ from $120^{\circ}$, and where $d(\theta)$ is the distance

(a)

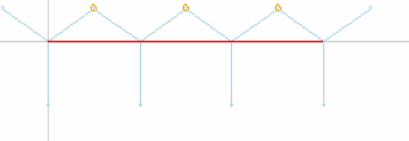

$120^{\circ} / 120^{\circ} / 120^{\circ} \times 1$ (b)

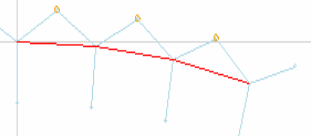

$115^{\circ} / 115^{\circ} / 115^{\circ} \times 1$ (c)

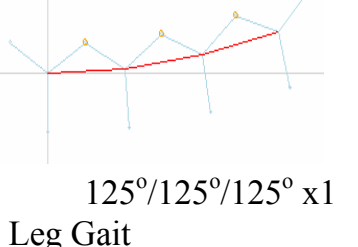

traveled by the center in one rotation $d(\theta)=2 l_{e} \operatorname{Sin} \beta \operatorname{Sin} \frac{\theta}{2}$. We can invert this to get an expression for the three leg gait angle in terms of the desired radius of curvature:

$$
\tan \theta / 2=\frac{r_{c} \sqrt{3}}{2 l_{e} \operatorname{Sin} \beta+r_{c}}
$$




\subsection{Compound Gaits.}

Compound gaits are gaits composed of two or more simple gaits. There are compound gaits that can be used for alternate patterns of area coverage; for example, the compound gait $g c 2$ consisting of $g l=[(L 1,100)(L 2,100)]$ and $g 2=[(L 2,100)(L 3,100)]$ produces an unusually complicated pattern (Fig. 10). There are two useful compound gaits that can be produced from the three leg simple gaits however, and we concentrate on these in this paper. They are: cycloid gait and orienting gait.

Cycloid Gait. A disadvantage of the straight line three leg gait is that it requires the mechanism to rotate by $120^{\circ}$ on each leg. It may be difficult to reach this level of performance on a real platform. The current rotopod prototype, described in more detail in [6], is limited to about $20^{\circ}$ by mechanical and experimental constraints. While we expect this could be increased substantially, we expect reliable $120^{\circ}$ rotations might be difficult to achieve. The cycloid gait, so named because it resembles a prolate cycloid, offers an approach to forward motion without the large angle rotation. The cycloid compound gait consists of two three leg constant angle simple gaits, the first with a large radius of curvature $R$ and the second with a smaller radius $r$. We use $R=2.7 r=39 \mathrm{~cm}$ in this paper, see Fig. 11 , as that produces an integral number of leg rotations in each half circle. The cycloid gait is clearly not as efficient as the straight line three leg gait from the perspective of forward motion. The straight line gait moves the robot forward $\sqrt{3} l_{e} \operatorname{Sin} \beta$ or $24 \mathrm{~cm}$ on each leg. In 9 leg rotations the cycloid gait move the center forward $2 r(2.7-$ 1) $=49 \mathrm{~cm}$; 9 rotations in straight line gait would be $216 \mathrm{~cm}$.

Note however that the cycloid gait covers more area than a straight line

Figure 10: Gait $g c 2 \times 4$ gait would cover. From the perspective of a search or surveillance applications, the cycloid gait may indeed be a good balance between point-to-point

locomotion and area coverage locomotion. To illustrate this point, we can calculate a fractal dimension for the cycloid gait.

Fractal dimension [12] is defined as the log of the number of repetitions divided by the $\log$ of the scaling factor for the repetitions. Consider the cycloid gait defined by $R=a r$ for a constant $a$. The distance traveled by the center is the sum of the two half circumferences or $\pi(a+1) r$. Let us consider populating the circumference with a smaller cycloid defined by

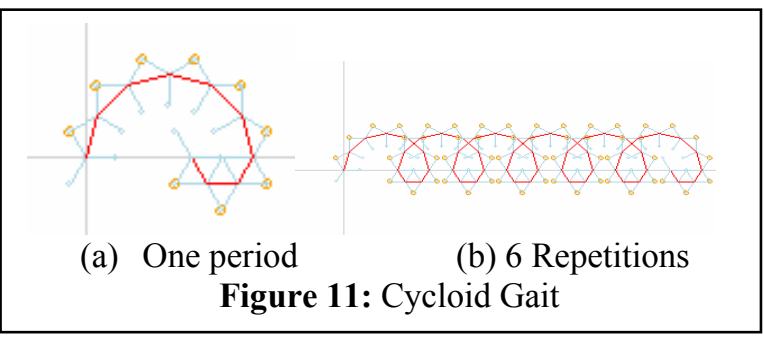

$b r^{\prime}=r$ and $b R^{\prime}=R$. The number of such smaller cycloids needed is $\frac{\pi b(a+1)}{2 a}$. In our case, $a=2.7$ and we select a magnification of $b=a=2.7$. The fractal dimension is then $\log (5.81) / \log (2.7)=1.7$. We can compare this to the fractal dimensions of some other well known fractals: The fractal dimension of the Koch curve, for example, is 1.26, and for the Sierpinski gasket is 1.58 .

Orienting Gait. This compound three leg gait allows us to approximate a separation of the orientation of the mechanism from the translation of the mechanism. For example, to walk the mechanism around the four sides of a square using the straight line three leg gait (as in [6]), the mechanism needs to be rotated by $90^{\circ}$ on each corner. The mechanism will be rotated to an angle $\theta$ by $n$ repetitions of the gait $g r=[(L 1, \theta / 3 n),(L 2, \theta / 3 n),(L 3, \theta / 3 n)]$. The larger $n$ the better the precision with which the mechanism can attain the desired orientation.

\section{Trajectory Planning}

The rotopod mechanism offers a number of locomotion styles, and we present and compare three here:

1. Straight line locomotion using a three leg straight line gait and a three leg orienting gait.

2. Semicircular locomotion using a three leg curved gait and a three leg orienting gait.

3. Straight line locomotion using a three leg cycloid gait with $R=2.7 r$ and a three leg orienting gait. 
The input to the trajectory planning algorithm is a sequence of 2-D points. The objective is to produce a sequence of gaits that place the mechanism at each of the points in succession. All three styles were planned using the same basic algorithm, as follows:

1. For each point $p$ in the sequence :

2. Determine the angle $\varphi$ between the current rotopod orientation and the straight line joining the center of the rotopod to $p$.

3. Rotate by $\varphi$ (using a value of $n=2$ )

4. Calculate the distance $d$ between the current position and $p$.

5. Select either:

a. A number of repetitions of a straight line gait to cover $d$

b. A number of repetitions of a cycloid gait to cover $d$

c. A curved gait with radius of curvature $d / 2$.

6. Move to $p$ and measured the error.

In step 5, the gait was not restricted to complete all three leg rotations, it could end after the first or second, for better precision.

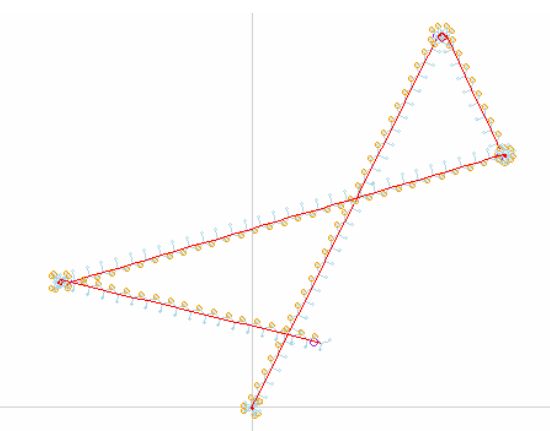

(a) Straight Line path

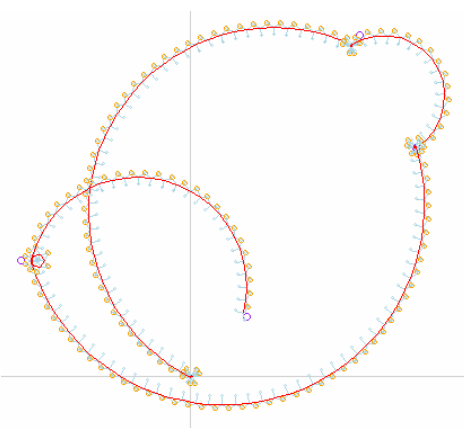

(b) Semicircular path

Figure 12: Trajectory planning using three locomotion styles.

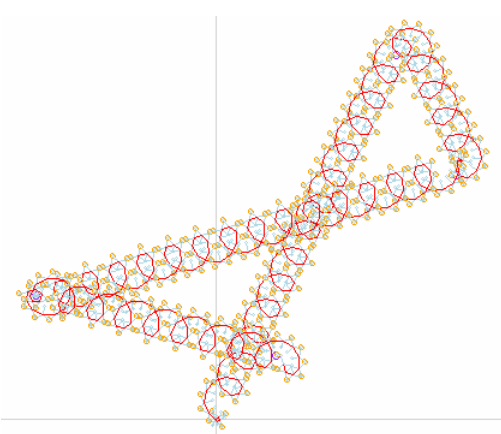

(c) Cycloidal path

The results are shown in Figure 12 (a) through (c) corresponding to the locomotion styles of 1 through 3 above.

The trajectory being tracked in Fig. 12 is $[(300,600),(400,400),(-300,200),(100,100)]$. The average mean squared sum of error (error in $x$ squared plus error in $y$ squared averaged for the path length) was 128 for the straight line path, 255 for the semicircular path, and 303 for the cycloidal path. The two components of the error were

1. the overstep on the last rotation of a gait, and

2. the precision of the orientation step.

Reducing either of these will reduce the error: for example, $n=3$ for the orienting gait will reduce the semicircular path error to 206.

\section{Discussion}

In this paper we have reviewed the rotopod mechanism first introduced by Lyons and Pamnany in [6], and we introduced a taxonomy of gaits for the robot as well as initial results for trajectory planning. The mechanism has an extremely rich vocabulary of possible gaits. The results of this analysis of gaits can be summarized as follows:

1. Straight line fast motion is possible, but requires large leg rotations.

2. Equal angle three leg rotations move the mechanism around the circumference of a circle.

3. Straight line slower motion is also possible using a cycloid gait, requiring smaller leg rotations.

4. The cycloid gait covers area effectively. The $R=2.7 r$ cycloid gait has a high fractal dimension of 1.7.

5. Two leg gaits can be used for spot maneuvers such as sharp turns. 
6. Two and three leg gaits can be used to cover an area with an increasing fine search pattern. Compound gaits can be used to cover non-annular search areas or for different scan patterns.

Trajectory generation algorithms were developed for straight line fast motion, for motion along semicircles and for straight line cycloidal motion, and an example of each following the same path was shown. A simple, common algorithm was used for all three. One important improvement would be to use an algorithm that does not treat orientation and translation as separate steps. There a number of papers related to trajectory planning for car-like robots that can easily be employed here, e.g., [5,4].

We believe this platform is well suited to search and reconnaissance applications: It has the potential for energy efficient motion, its natural locomotion style favors area coverage, and it has a compact and simple form. We can envisage future versions of the rotopod being telescoped and folded like a camera tripod for ease of carrying and deployment by soldiers or by larger robot platforms, and unfolded and deployed on an as-needed basis.

Future work will concentrate in two areas: a more effective prototype with which to conduct experimentation and the evaluation of one the projected key strengths of this platform, the efficiency with which it can search a designated area (e.g., [2]).

\section{References}

1. Birch, M., Quinn, R., Hahm, G., Phillips, S., Drennan, B., Fife, A., Beer, R., Yu, X., Garverick, S., Laksanacharoen, S., Pollack, A., Ritzmann, R., A Miniature Hybrid Robot Propelled by Legs. Proceedings IROS 01, Maui, Hawaii, Oct. 29. - Nov. 3, 2001.

2. Mei, Y., Lu, UH., Hu, C., and Lee, CSG. Energy-Efficient Motion Planning for Mobile Robots. Proceedings of the International Conference on Robotics and Automation, New Orleans LA, 2004.

3. Halme, A., Leppanen, I., Montonen, M., Ylonen, S., Robot Motion by Simultaneous Wheel and Leg Propulsion. Proc. 4th Int. Conference on Cliimbing and Walking Robots (CLAWAR), 2001.

4. Hwang, J-H., Arkin, R.C., Kwan, D-S., "Mobile Robots at your fingertips: Bezier Curve On-line Trajectory Generation for Supervisory Control” Proc. IROS 2003 Oct. 27-31 Las Vegas Nevada 2003.

5. Latombe, J.-C. Robot Motion Planning. Norwell, MA: Kluwer Academic Publishers, 1991.

6. Lyons, D., and Pamnany, K., Rotational Legged Locomotion. Proc. ICAR 2005, July 18-20 ${ }^{\text {th }}$, Seattle WA 2005.

7. Pamnany, K., An Analysis of Motion Strategies and Energy Efficiency for a Tripedal Robot. Fordham Univ. Dept. of Computer \& Information Science. MS Thesis. May 2005.

8. Quinn, R. D., Nelson, G.M., Bachmann, R.J., Kingsley, D.A., Offi, J., and Ritzmann, R. E., (2001). Insect Designs for Improved Robot Mobility. Proc. of Climbing and Walking Robots Conference (CLAWAR01), Professional Engineering Publications, ed. by Berns \& Dillmann, Karlsruhe, Germany, pp. 69-76.

9. Saranli, U., Buehler, M., and D. E. Koditschek, Design, Modeling and Preliminary Control of a Compliant Hexapod Robot, IEEE Int. Conf. Robotics and Automation, San Francisco, California, April 2000.

10. Steeves, C., Buehler, M., Penzes, S.G., Dynamic behaviors for a Hybrid Leg-Wheel Mobile Platform. Proc. SPIE, 2002, Vol. 4715, pp 75-86, Orlando, April 2-3, 2002.

11. Talebi, S., Poulakakis, I.,. Papadopoulos, E, and Buehler, M., Quadruped Robot Running with a Bounding Gait, Int. Symp. Exp. Robotics, Honolulu, HI, Dec 2000.

12. Theiler, J. Estimating fractal dimension, Journal of the Optical Society of America A - Optics and Image Science, 7(6), (1990) 1055-1073. 